\title{
Blucher
}

\author{
Blucher Proceedings \\ Cuba e Brasil no Século XXI (CBS21) \\ Desafíos Estratégicos e Institucionais da Inovação
}

\section{Cuba y Brasil: algunas propuestas de carácter estratégico}

\author{
Dr. Hugo Pérez Rojas ${ }^{1}$, Dr. Helio T. Coelho ${ }^{2}$ \\ y Dr. César Augusto Zen Vasconcellos ${ }^{3}$
}

\section{Introdução}

Este ensayo, que se presenta a los lectores, es el resultado de un breve estudio con el objectivo de contribuir a la consolidación de una asociación entre Cuba y Brasil. En este sentido, presentamos propuestas que buscan la posible asociación de académicos, empresarios y gobernantes con el objectivo de la realización de una nueva economía de carácter social que puede, por un lado, fortalecer la capacidad productiva de estas naciones hermanas, y por el otro, materializar nuestros sueños de una sociedad justa y fraterna que protege el medio ambiente y busca la sostenibilidad. La decisión de dar prioridad a las alianzas de Cuba con los estados del noreste de Brasil se debe a las similitudes entre estas regiones, ya sea por el clima, la cultura, la comida, la geografía, la economía, la producción animal, o porque los suelos de estas regiones son adecuados para la siembra de caña de azúcar y para la producción de destilados y del tabaco, y incluso para la producción de bioenergía, entre otros aspectos. Proponemos estas ideas, aunque en una etapa preliminar de desarrollo teórico, como una propuesta de discusión que requerirá, para su logro más definitivo y completo, debido a su complejidad, la participación de pensadores e investigadores de muchas distintas áreas del conocimiento y la decisión y la

\footnotetext{
${ }^{1}$ Instituto de Cibernética, Matemática y Física - ICIMAF, La Habana, Cuba. E-mail: hugo@icimaf.cu.

${ }^{2}$ Membro da Academia Brasileira de Ciências - ABC y de la Academía de Ciencias de Pernambuco - ACP, Recife, Brasil. E-mail: heliotcoelho@terra.com.br.

${ }^{3}$ Universidade Federal do Rio Grande do Sul - UFRGS, Porto Alegre, Brasil; International Center for Relativistic Astrophysics Network - ICRANet, Pescara, Itália. E-mail: cesarzen@cesarzen.com.
} 
participación de autoridades gubernamentales de ambos países. Y además, para lograr sus objetivos es esencial tener una participación efectiva en las propuestas de la Universidad Federal de Pernambuco (UFPE), de la Universidad Rural de Pernambuco (UFRPE), de la Universidad Federal de Alagoas (UFAL) y de la Universidad Federal de Paraíba (UFPB) en colaboración con las universidades e instituciones de enseñanza e investigación en general de Cuba, como por ejemplo, la Universidad de Habana (UH) y el Instituto de Cibernética, Matemática y Física (ICIMAF). Presentamos a seguir algunas de estas propuestas.

\section{Renovación y Revitalización Urbana para la Innovación y la Creatividad}

Esa es una propuesta de creación de un proyecto de espacios urbanos inteligentes que involucra los proyectos ya existentes de renovación y revitalización urbana de las ciudades de La Habana, Recife, Olinda, João Pessoa y Maceió (véase el Apéndice A) para la innovación y la creatividad.

El vertiginoso crecimiento de los espacios urbanos, desde un punto de vista sociológico y demográfico, genera nuevos escenarios que han impulsado, en los campos de la economía, de la política y del medioambiente, el concepto de ciudades inteligentes o espacios urbanos inteligentes (véase el Apendix B). El reto de la gestión de estos espacios, extremadamente complejos, requiere una nueva mirada y acciones que hacen uso de los avances tecnológicos para mejorar la calidad de vida de sus habitantes. En realidad, este concepto tiene múltiples matices e interpretaciones. La asociación entre las ciudades mencionadas anteriormente presenta, en nuestra opinión, una oportunidad para construir una nueva realidad socioeconómica de Cuba y de la región noreste de Brasil.

Sabemos que tanto Recife, Olinda, Maceió y João Pessoa, como La Habana ya tienen proyectos para recuperar su patrimonio histórico. El objetivo de esta propuesta es la asociación entre los proyectos ya existentes de la renovación urbana y del patrimonio histórico y la revitalización de estas ciudades con propuestas integradas para la atracción de inversiones en el campo de la economía creativa, a través de la creación de Polos Integrados de las Industrias Creativas y de la Economía Creativa basada en las alianzas estratégicas que cruzan la educación, la creatividad, las artes, la cultura, las actividades comerciales, la ciencia, la tecnología y la innovación (véase el Apéndice C).

Para lograr la propuesta se tratará de profundizar las relaciones y la colaboración entre La Habana, Recife, Olinda, João Pessoa y Maceió con sus entornos creativos y productivos, a través de la formulación de proyectos de asociación entre las instituciones de Ciencia y Tecnología (ICT) de los dos países, así como con los Núcleos de Innovación Tecnológica (NIT), las Empresas de Base Tecnológica 
(EBT) (véase el Apéndice C), los creadores, los inventores, los investigadores independientes y los Parques de Ciencia y Tecnología, las incubadoras, las organizaciones civiles y sus gobiernos municipales. La propuesta tiene como objetivo, además la participación de los empresarios de las distintas ramas de actividades, con sede en Brasil, en Cuba y incluso en otros países, estimulando así la formación de alianzas estratégicas con miras a un desarrollo socioeconómico sostenible de esas regiones urbanas.

\section{Asociación entre los Puertos de Mariel y de Suape}

Esa asociación también puede contribuir eficazmente a la realización de una nueva realidad económica en Cuba y en la región noreste de Brasil. Y Brasil además, como el socio principal en la construcción del puerto de Mariel (véase el Apéndice D) podría, a través de propuestas como esta que presentamos, seguir contribuyendo a la inserción efectiva del puerto de Mariel, entre los programas de desarrollo más audaces y avanzados ya proyectados por una nación.

La construcción de una asociación entre los Puertos de Suape (véase el Apéndice E) y de Mariel puede impulsar el mercado de los grandes barcos comerciales y cruceros en el Caribe y en la América del Sur, así como promover los intereses comunes de los puertos asociados, y además representar los intereses y preocupaciones de los gobiernos de Brasil, Pernambuco y Cuba para la tomada de decisiones de gestión y geopolíticas.

El alcance de la propuesta amplía las áreas geográficas del Caribe y de la América del Sur con el fin de promover actividades comerciales eficientes, así como estrategias comunes de gestión y de negocios marítimos, además de la formación del personal que opera en los puertos, reduciendo al mínimo los daños y desastres ambientales.

Esta alianza también podrá establecer directrices comunes para el atraque de los buques con el fin de eliminar los riesgos, promover intercambios de experiencias y conocimientos dirigidas a mejorar el funcionamiento de las tecnologías de los dos puertos, y inclusó promover la creación de un Parque y de una Universidad de Ciencia y Tecnología del Mar.

Un aspecto de la alianza estratégica importante será el área de los negocios, lo que permitirá el establecimiento de decisiones conjuntas con miras a la instalación de empresas de importancia estratégica en sus instalaciones con el fin de aumentar la competitividad de los dos puertos y de los operadores asociados, así como la promoción del desarrollo social y económico de las regiones donde se ubican, las regiones de Recife, Maceió y João Pessoa, en Brasil, e la región del Mariel, en Cuba. 


\section{Creación de una Réplica del Puerto Digital en la ZEDM}

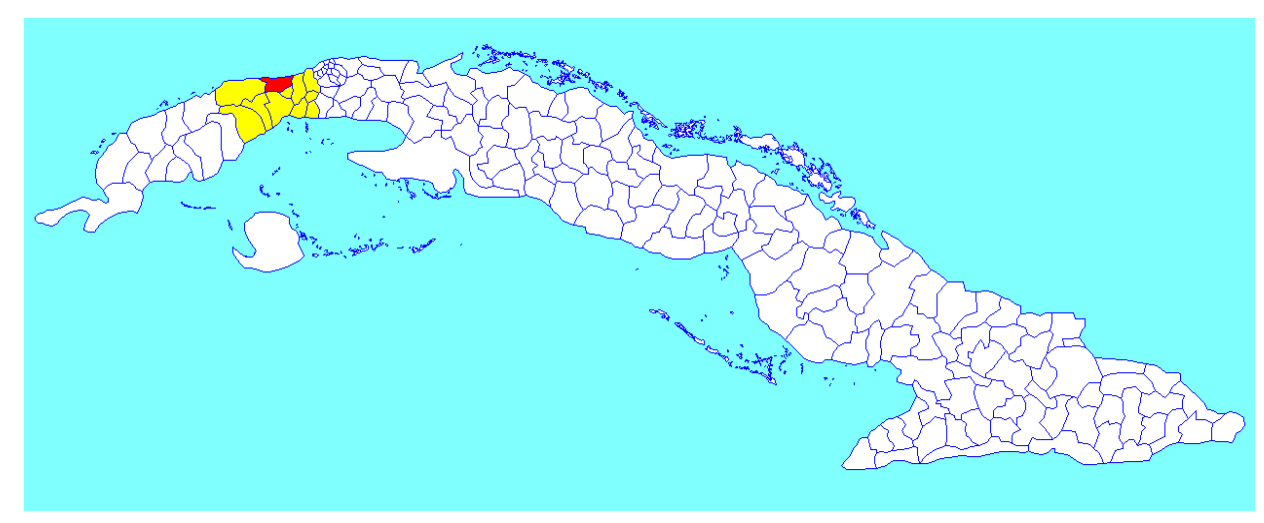

Figura 1: Ubicación del puerto de Mariel y de la ZEDM en Cuba (imagen sin copyright.).

" Con la infraestructura de la Zona Especial de Desarrollo del Mariel - ZEDM, se inició el cumplimiento del lineamiento del Gobierno Cubano de promover la creación de Zonas Especiales de Desarrollo que permitan incrementar la exportación, la sustitución efectiva de importaciones, los proyectos de alta tecnología y desarrollo local; y que contribuyan con nuevas fuentes de empleo. No obstante, las normativas que la concretan, y el propio desarrollo de la ZEDM son transversales a la mayoría de los lineamientos referidos a la inversión extranjera, por no decir todos. Será, además, un polígono de ensayo para el establecimiento de otros espacios de este tipo en la Isla. Aunque la zona es especial en el sentido franco de la palabra, el término también se refiere a un grupo de tratamientos y regímenes dirigidos a hacer lo más atractiva posible la participación de empresas extranjeras y nacionales, con beneficios e incentivos particulares y bastante redituables. Sus concesionarios y usuarios dispondrán de un tratamiento aduanero especial, así como regímenes favorecedores en las áreas tributarias, de seguros, y monetaria y bancaria, entre otras. El desarrollo de la ZEDM mucho dependerá de las gestiones, la capacidad y la innovación de las instituciones encargadas. Y de la sabiduría, habilidad y emprendimiento de los inversores extranjeros y nacionales. Mariel les abre una oportunidad única: un nicho donde se ofrecen las ventajas que caracterizan a estos espacios en cualquier lugar, pero con el valor agregado de estar en un país que será estratégico en el mapa del transporte marítimo y el comercio global tras concluirse la ampliación del Canal de Panamá en 
2015, y con un capital humano de altísima calificación técnica y una voluntad política a todo prueba para que gane el país y quienes tengan la luz larga de aprovechar la oportunidad y asentar negocios en la Zona Especial de Desarrollo de Mariel."

Con estas palabras se desprende la importancia estratégica de la ZEDM. Y una asociación de la ZEDM con el Porto Digital de Recife (véase el Apéndice F), por su propia naturaleza, puede contribuir de manera decisiva a transformar la realidad económica de Cuba y del noreste de Brasil.

Así, el objetivo de la propuesta es crear una réplica del Puerto Digital de Recife en la ZEDM, una ubicación estratégica en el Caribe, que se encuentra 367 kilómetros del Puerto de Miami en una asociación de instituciones públicas y privadas de Cuba y Brasil: empresas, industrias, universidades y gobiernos, promoviendo así los cambios económicos y sociales con el fin de generar riqueza, empleo e ingresos en Cuba y en Pernambuco. La creación de esta replica en la ZEDM expandirá el potencial comercial de las 200 empresas participantes en el puerto digital, poniéndolos en las fronteras del mayor mercado consumidor mundial. Por otra parte, el establecimiento de esas empresas en la región de Mariel impulsará la creación de empresas innovadoras cubanas para acelerar el proceso de actualización del modelo económico de Cuba.

\section{Apéndice A}

\section{La Habana}

Es la capital de la República de Cuba, su ciudad más grande y es el más importante centro económico y cultural del país. La Habana es la sede oficial de los órganos rectores de los poderes legislativo, ejecutivo y judicial del Estado, de todos los organismos centrales y de casi todas las empresas y asociaciones del país. También cuenta con el mayor número de sucursales y es la sede de las entidades extranjeras arraigadas en Cuba. También es conocida como la "Villa de San Cristóbal de La Habana", así como los apellidos "Llave del Nuevo Mundo"y "Ciudad de las Columnas" (según el escritor cubano Alejo Carpentier"5). Administrativamente la provincia de La Habana comprende el territorio de la ciudad, los pueblos cercanos y una zona rural fronteriza. Fue fundada el 16 de noviembre de 1519 por el conquistador español Diego Velázquez de Cuéllar. Sus edificios históricos han sido declarados Patrimonio de la Humanidad por la UNESCO. La Habana cuenta con alrededor de 2.4 millones de habitantes.

\footnotetext{
"Extractado de "Mariel, el camino más corto", René Tamayo, Diario de la Juventud Cubana (www.juventudrebelde.cu/cuba/2013-11-02/mariel-el-camino-mas-corto).

${ }^{5}$ Véase por ejemplo www.cervantes.es/.
} 


\section{Recife}

Es la capital del Estado de Pernambuco, Brasil. Con una superficie aproximada de $217 \mathrm{~km}^{2}$, se encuentra a orillas del Océano Atlántico, y tiene una población de 1,546,516 personas. Metrópoli más rica en el noreste de Brasil, juega un papel importante en la región, que alberga un gran número de oficinas regionales, de instituciones nacionales y empresas públicas y privadas, con un área de influencia que abarca otras capitales, como João Pessoa, Maceió, Natal y Aracajú. Recife fue elegida como una de las ciudades con economías más desarrolladas de los mercados emergentes en el mundo. La ciudad se destaca por tener el mayor parque de tecnología en Brasil, el Puerto Digital, el mayor número de consulados extranjeros fuera del Rio-São Paulo, el segundo mayor centro médico de Brasil, el mejor aeropuerto de Brasil, grandes centros comerciales, el mayor PIB per cápita y el mayor ingreso per cápita entre las capitales del noreste de Brasil, el noveno mayor número de rascacielos en las Américas, sólo superada por Nueva York, São Paulo, Rio de Janeiro, Toronto, Buenos Aires, Ciudad de México, Chicago y Caracas, un fuerte sector de la construcción, un mega Complejo Industrial y Portuario, SUAPE, que alberga el mayor y mejor puerto en Brasil. Con un gran potencial para el turismo y la fuerte vocación para el turismo de negocios, Recife a menudo es elegida como sede de varios eventos, como simposios, conferencias y congresos. La Universidad Federal de Pernambuco - UFPE, y la Universidad Federal Rural de Pernambuco UFRPE, en particular, son dos de los centros de generación de conocimiento más importantes en la región y en el país.

\section{Olinda}

Es una de las mejor conservadas ciudades coloniales de Brasil. Olinda se encuentra ubicada en la Región Metropolitana de Recife. Tiene una población de 375.559 habitantes. Fue declarada Patrimonio Histórico y Cultural de la Humanidad por las Naciones Unidas para la Educación, la Ciencia y la Cultura (UNESCO) en 1982. Además de su belleza natural, Olinda es también uno de los centros culturales más importantes de Brasil.

\section{João Pessoa}

Es la capital del Estado de Paraíba, Brasil. Es conocida como la "Puerta del Sol", debido al hecho de estar situado en el punto más oriental de las Américas, lo que hace que la ciudad sea conocida como el lugar "donde el sol sale por primera vez en las Américas". Fundada en 1585 bajo el nombre de Nuestra Señora de las Nieves, la ciudad de João Pessoa es la tercera capital más antigua del Brasil. En João Pessoa hay un complejo parque industrial, que consiste en varios segmentos: alimentos, automotriz, bebidas, bentonita, cemento, hormigón, cuero, metal, mu- 
ebles, vidrios, papel, suelos de cerámica, química, textil, informática, entre otros. João Pessoa tiene el mayor parque industrial en el Estado de Paraíba, destacando algunas industrias de renombre internacional como Ambev, Coca-cola, Euroflex, Coteminas, British American Tobacco, Penalty. João Pessoa, con sus playas es un importante centro para el turismo nacional. La Universidad Federal de Paraíba - UFPB, en particular, es uno de los centros de generación de conocimiento más importantes en la región y en el país.

\section{Maceió}

Es la capital del Estado de Alagoas, Brasil. Es el mayor productor brasileño de sal de roca. Su sector industrial se compone de diversos productos químicos, azúcar y alcohol, cemento y alimentos. Su economía también incluye la agricultura y la extracción de gas natural y petróleo. Conocida como "Ciudad-Sonrisa"y "Paraíso de las Aguas", ahora es considerado como el "Caribe brasileño", debido a su belleza natural, que atrae a turistas de todo el mundo. La ciudad cuenta con muchos establecimientos industriales y en particular con un polo cloro-químico.

\section{Apéndice B}

\section{Ciudades inteligentes,espacios urbanos inteligentes ${ }^{6}$}

El término ciudad inteligente, o espacio urbano inteligente, puede tener significados diferentes:

- Las ciudades o zonas urbanas donde ocurren de manera intensa las actividades de carácter virtual. El término puede ser utilizado como el equivalente de la ciudad digital, de ciudad de la información, de una ciudad conectada, tele ciudad, ciudad basada en el conocimiento, comunidad electrónica, entre otros, abarcando así una amplia gama de aplicaciones en los campos de la electrónica y digital.

- Las ciudades o zonas urbanas donde hay un desarrollo social e económico inteligente. Es decir, un tipo de desarrollo que se basa en las tecnologías modernas de la información y de la comunicación. En este contexto, las comunidades que ocupan estos espacios urbanos se llaman "comunidades inteligentes", ya que desempeñan en forma consciente y coordinada, — a través de las tecnologías de la información y de la comunicación --, la transformación de la vida, y la organización del ocio y de las distintas formas y potencialidades del trabajo en los territorios que ocupan.

\footnotetext{
${ }^{6}$ Este texto es una adaptación de varios otros textos. Para una comprensión más completa de estas cuestiones véase por ejemplo el sitio web: http://www.intelligentcommunity.org/.
} 
- Las ciudades y entornos urbanos que crean por su vez entornos interactivos que encarnan el mundo de las comunicaciones produciendo la transferencia de las potencialidades virtuales al mundo físico. En este contexto, las ciudades inteligentes o espacios urbanos hacen referencia a un entorno físico en el que las tecnologías de comunicación e información están incorporadas en los objetos físicos y en los ambientes en los que vivimos.

- Los territorios que incluyen los sistemas de las TIC para la innovación y para el logro de la economía creativa. En estos entornos, es necesario combinar, en el contexto de la sociedad del conocimiento, amplios mecanismos y herramientas de la información y la comunicación, por ejemplo, "banda ancha" en las escuelas, en las empresas, en los edificios públicos, en las viviendas, con el objetivo de mejorar la educación y las actividades de la fuerza laboral, así como para establecer políticas y crear programas que promuevan la democracia digital, la reducción de las brechas digitales. Y además para asegurar que todos los sectores de la sociedad y sus ciudadanos puedan beneficiarse de los frutos de la innovación en las iniciativas públicas y privadas con el objectivo de crear grupos económicos con alto valor añadido, y posibilitar la existencia de "capital de riesgo" para apoyar el desarrollo de nuevos proyectos empresariales, sociales y culturales.

- Los territorios caracterizados por alta capacidad de aprendizaje y de la innovación de su gente y de sus instituciones involucradas en la generación de nuevos conocimientos y que disponen de infraestructura digital para la comunicación y para la gestión del conocimiento. La característica distintiva de una ciudad o área urbana inteligente es el alto rendimiento en el campo de la innovación, ya que la innovación y la búsqueda de la solución de los nuevos problemas que plantean las sociedades modernas son las características distintivas de la "inteligencia de una ciudad o espacio urbano".

\section{Apéndice C}

\section{Las industrias creativas, la economía creativa ${ }^{7}$}

- Las industrias creativas: se refieren a las industrias que tienen su origen en la creatividad, las habilidades y el talento con gran potencial de creación de riqueza a través de la generación y explotación de la propiedad intelectual. Las industrias creativas se refieren a una amplia gama de actividades económicas

\footnotetext{
${ }^{7}$ Este texto es una adaptación de varios otros textos. Para una comprensión más completa de estas cuestiones véase por ejemplo el sitio web: http://creativecities.britishcouncil.org/creative-industries/.
} 
que están orientadas a la generación o la explotación de conocimientos e información. Son la base de la economía creativa.

- Economía Creativa: el término incluye una serie de actividades tales como publicidad, arquitectura, arte, artesanía, diseño, moda, cine, música, artes escénicas, edición, investigación y desarrollo (I\&D), software, juegos y juguetes, televisión y radio, los juegos de video, las actividades comerciales, el desarrollo industrial y comercial a través de la producción científica y tecnológica innovadora y patentes de negocios. Las industrias creativas se basan principalmente en la creatividad humana, el recurso económico más importante de la Sociedad del Conocimiento. Es importante destacar que, en la Sociedad del Conocimiento, a través de la inserción de conocimientos en productos y procesos industriales, bien como en las actividades de comercio y de servicios, esta nueva forma de organización social ha redefinido los conceptos de productividad de las economías nacionales y los activos de las empresas en todo el mundo sobre la base de una redefinición del papel del conocimiento como un elemento transformador de los sistemas de producción. Y en este sentido el conocimiento ocupa hoy el papel más importante en la reconfiguración de los diferentes sistemas socioeconómicos y productivos, por medio de la creación de nuevos patrones de producción y comercialización, influyendo decisivamente en la relación capital-trabajo y en el aumento de las posibilidades para el bienestar y la calidad de vida de los ciudadanos. En este contexto, las industrias del siglo 21 dependerán cada vez más en la creatividad y en la innovación, con énfasis en la competitividad empresarial y en la creación de entornos propicios para el desarrollo de nuevas patentes.

- Innovación: la palabra "innovación" viene del latín, innovare, y tiene el significado stricto de "novedad"o "renovación". Innovación es el resultado de un proceso que implica, a través de la investigación científica y tecnológica, o incluso de una actividad no académica, en la creación, invención, evolución, aplicación e implementación económica de nuevos productos, nuevas ideas, nuevas propuestas, o mejoras en la calidad en los procesos, productos, procedimientos, o servicios ya existentes en el campo enpresarial o no, para ampliar la capacidad de comercialización y competitividad de las empresas de los países.

- Instituto de Ciencia y Tecnología (ICT): agencia gubernamental o privada, directa o indirectamente activa en el campo del conocimiento científico y tecnológico, así como universidades e instituciones de investigación, empresas públicas y privadas, que tienen misión institucional de llevar a cabo actividades relacionadas con la innovación y la investigación en ciencia y tecnología 
en entornos de producción para las actividades comerciales, industriales y/o de servicios.

- Núcleo de Innovación Tecnológica (NIT): miembro de la estructura de los Institutos de Ciencia y Tecnología con el objetivo de la gestión de la política y la innovación institucional y la protección de la propiedad intelectual.

- Empresa de Base Tecnológica (EBT): una empresa legalmente constituida cuya actividad productiva está dirigida al desarrollo o mejora de productos, procesos o actividades de servicios basados en la aplicación sistemática de conocimientos científicos y tecnológicos.

- Creación: invención, modelo de utilidad, diseño industrial, programa de ordenador, topografía de circuitos integrados, cultivo de nuevas variedades o cualquier otro desarrollo tecnológico, creado por uno o más creadores que generan o pueden generar, refinar o mejorar procesos, productos o servicios de las actividades empresariales.

- Novedades de Patentes: novedades de patentes son el requisito previo para la existencia de una creación y por lo tanto, son absolutamente imprescindibles para la concesión del derecho exclusivo de una patente.

- Creador: investigador que actúa como un inventor, un mentor, un artista o autor del proceso de creación.

- Investigador público: los ocupantes efectivos de la función pública, cuyas tareas funcionales son la investigación básica o aplicada en las áreas de innovación, de la ciencia y de la tecnología en una Institución de Ciencia y Tecnología, así como los estudiantes regularmente matriculados y los profesionales que trabajan en la investigación científica y/o tecnológica.

- Inventor Independiente: las personas que no tienen cargo público o posición institucional, pero actúan, como un inventor, un mentor, diseñador, artista o autor del proceso de creación.

- Parque Científico y Tecnológico: una organización gestionada por profesionales especializados, cuyo objetivo principal es incrementar la riqueza de la comunidad mediante la promoción de una cultura de innovación y la competitividad de las instituciones relacionadas con la organización intensiva del conocimiento, como las universidades y los institutos de investigación. Un Parque Científico y Tecnológico implica necesariamente la promoción de la relación entre las universidades e instituciones de investigación con el sector empresarial y con la industria para estimular los procesos de innovación, facilitando la transferencia de tecnología y conocimiento entre la academia y 
el sector empresarial, promoviendo así el desarrollo sostenible en la región donde operan.

- Incubadora de Empresas: las organizaciones que apoyan la creación y el desarrollo de las micro y pequeñas empresas, industrias o áreas de servicio, basadas en la tecnología o la industria ligera, a través de la provisión de infraestructura básica, la mejora de las habilidades y técnicas y la capacidad de gestión de los empresarios y de manera complementaria, para facilitar su acceso a la innovación tecnológica y la entrada en el mercado.

\section{Apéndice D}

\section{Puerto de Mariel}

La ampliación del puerto de Mariel, a 45 kilómetros de la capital cubana, regresa al primer plano de la política internacional con las inversiones brasileñas alrededor de U\$D 802 millones en bienes y servicios, en una primera etapa, para la ampliación del Puerto de Mariel, con la participación en este proyecto de más de 400 empresas brasileñas. En una segunda fase, el Brasil invertirá más 290 millones de dólares en la Zona Especial de Desarrollo del Mariel. Brasil quiere convertirse en socio económico de primer orden de Cuba al aumentar el flujo de comercio bilateral entre los dos países ${ }^{8}$.

Con la ampliación del Canal de Panamá, la ubicación estratégica del puerto de Mariel, en la región del Caribe despierta el interés de convertir al puerto del Mariel en un gigantesco centro de almacenamiento y manejo de carga marítima regional. En realidad, la expansión del puerto de Mariel está relacionada directamente con la modernización del canal de Panamá que para 2015 permitirá el paso por sus compuertas de mega-cargueros de profundo calado ${ }^{9}$ que cruzarán en ambas direcciones el Pacífico, el Atlántico y el Caribe. Cuba tiene la intención de convertirse, con el puerto de Mariel y la Zona Especial de Desarrollo de Mariel (ZEDM), un centro logístico de mercancías regional e internacional.

\section{Apéndice $E$}

\section{Complejo industrial del puerto de Suape}

Ese complejo es considerado uno de los principales polos de inversiones en Brasil. El puerto de Suape tiene una estructura moderna, con profundidades entre

\footnotetext{
${ }^{8} \mathrm{http}: / /$ www2.planalto.gov.br/; www.youtube.com/watch? $v=s 8 a C g R 2 Q P$ Poo.

${ }^{9}$ En Mariel se está construyendo muelles donde podran atracar embarcaciones Súper-PostPanamax con calado de 18 metros.
} 
$15 m$ y $20 m$ y un gran potencial para la expansión. Su ubicación estratégica en relación a la navegación lo mantiene conectado a más de 160 puertos de los cinco continentes, con líneas directas a Europa, América del Norte y África.

El movimiento portuario de Suape crece a buen ritmo y consolida el complejo como puerto concentrador y distribuidor de cargos. En 2011, el manejo de carga fue de 11 millones de toneladas y de los contenedores fue de más de 400 mil unidades, lo que representa un crecimiento del $25 \%$ y $33 \%$, respectivamente, en comparación con el año anterior. Su diseño como un puerto industrial ofrece las condiciones ideales para la instalación de nuevas empresas y el desarrollo tecnológico en distintos segmentos. Suape tiene una infraestructura terrestre propia en constante desarrollo y modernización, conectado con ferrocarriles y carreteras ${ }^{10}$.

El puerto interior ha ganado recientemente cunas nuevas y, además, el complejo también incluye el suministro de gas natural, la electricidad, el agua cruda y agua tratada. Situado en la región metropolitana de Recife cuenta con una superficie de 13.500 hectáreas, distribuida en las zonas del puerto, servicios industriales y administrativas, preservación ecológica y conservación del patrimonio cultural. Su área de influencia abarca todo el estado de Pernambuco y parte de los estados de Alagoas y Paraíba. Hoy hay más de 100 compañías operativas en Suape, responsables de más de 25 mil puestos de trabajo directos y otras 50 empresas en construcción. Entre ellas, la industria química, metalúrgica construcción naval y la logística, lo que reforzará los polos de generación de energía, líquidos y gases a granel, los alimentos y la energía eólica, y espacios abiertos en otros segmentos industriales. Las inversiones suman más de 40 mil millones de dólares, generando 15,000 nuevos puestos de trabajo y más de 40 mil empleos en la construcción ${ }^{11}$.

\section{Apéndice F}

\section{Puerto Digital de Recife}

El Puerto Digital de Recife es un resultado del ambiente de innovación que se consolidó en Pernambuco, en las últimas décadas. En una región atractiva para la innovación, las instituciones, las empresas, las universidades y los gobiernos promovieron los cambios económicos y sociales que están generando riqueza, empleo e ingresos. El punto de partida de esta nueva economía es el Porto Digital, definido como el Arreglo Productivo de Tecnologías de la Información y la Comunicación y la Economía Creativa, que se encuentra en Recife, capital de Pernambuco, en el noreste de Brasil. En esta histórica ciudad, escenario de la segunda invasión

\footnotetext{
${ }^{10}$ Hasta la publicación de este artículo, la página web del puerto (www.suape.pe.gov.br) no se ha actualizado. Por lo tanto la información contenida en esta publicación se refiere al año 2011.

${ }^{11}$ Fuente de la información: sitio oficial del puerto de Suape - www.suape.pe.gov.br.
} 
holandesa en 1630, se escurre la mayor producción de azúcar en el Brasil colonial, por lo que durante siglos fue una de las ciudades más modernas e importantes del país. Hoy en día, Pernambuco se insinúa en el escenario mundial por su capital humano, el espíritu empresarial y la innovación. De los ingenios para una economía basada en los servicios, en las TIC y en la Economía Creativa.

En 2010, las 200 compañías ubicadas en el Puerto Digital tuvieran una facturación de USD 1 billón. En este contexto el conocimiento cambió la realidad de Pernambuco: producir localmente y exportar servicios de alto valor añadido para el mundo es el objetivo principal del Puerto Digital, un proyecto de desarrollo económico que cuenta con inversión pública y asociaciones del sector privado con las universidades, y que es basado en la formación de un sistema de innovación local que en la actualidad cuenta con 200 entidades entre empresas de las TIC, de la economía creativa, de servicios especializados y de agencias en general.

El barrio donde se encuentra el Puerto Digital, Santo Amaro, cuenta con una infraestructura adecuada para la instalación de empresas de TIC por tener una excelente infraestructura y servicios de telecomunicaciones. En 100 hectáreas de Recife se instalaron ocho kilómetros de fibra óptica y veintiséis de tuberías, por lo que la región sea una de las más modernas del país. Antecedentes Es importante señalar que ya existe un proceso de colaboración entre Cuba y Pernambuco. La cooperación entre los puertos de SUAPE y de MARIEL, para el establecimiento de rutas marítimas internacionales, fue uno de los aspectos concretos de cooperación establecidos durante una reunión en el mes de mayo de 2011, entre el gobernador del Estado de Pernambuco, Eduardo Campos y el embajador de Cuba en Brasil, Carlos Rafael Rodríguez Zamora. Entre los otros temas tratados por el Gobernador y el Embajador, cabe destacar el fomento de los flujos turísticos mediante la creación de líneas aéreas directas entre La Habana y Recife. En esta reunión, se discutieron otros temas. Uno de ellos fue la invitación del gobernador para la delegación olímpica cubana adoptar Pernambuco como lugar de preparación para los Juegos Olímpicos de Río de Janeiro en 2016. También se discutió un estudio sobre formas de exportar productos como el pollo, lácteos y ropa a Cuba y aumentar el intercambio de estudiantes y grupos culturales cubanos y brasileños.

Recife, Olinda, Maceió, 2014 